\title{
Políticas sociales "progresistas" en el Cono Sur: discursos y prácticas desde los Estados
}

\author{
Christian Adel Mirza \\ Universidad de la República (Udelar)
}

\section{Políticas sociales "progresistas" en el Cono Sur: discursos y prácticas desde los Estados}

Resumen: El cambio de orientación de las políticas sociales en América Latina, particularmente en el Mercosur, obedece al relevo de las fuerzas políticas que han accedido al ejercicio del poder político y el control de los principales resortes del Estado. Los cambios observados en el campo de las políticas públicas en el sentido de una mayor intervención del Estado, un abordaje diferente de la cuestión social y la prioridad colocada en los segmentos poblacionales en situación de vulnerabilidad socioeconómica, son algunas de las evidencias observadas. Y si bien son plausibles los resultados y logros, persisten rasgos del modelo residual lo que se manifiesta en contradicciones en el diseño y también en la implementación de políticas sociales; no obstante hay oportunidades para profundizar las transformaciones en curso. El artículo presenta - a partir de estudios empíricos y experiencias en la gestión a nivel regional - algunos aportes para el análisis de escenarios futuros.

Palabras clave: Políticas sociales. Izquierda. Estado.

\section{Políticas sociais "progressistas" no Cone Sul: discursos e práticas dos Estados}

Resumo: A mudança de orientação das políticas sociais em América Latina, particularmente no Mercosul, obedece ao relevo das forças políticas que tiveram acesso ao exercício do poder político e o controle dos principais recursos do Estado. As mudanças observadas no campo das políticas públicas no sentido de uma maior intervenção do Estado, de uma abordagem diferente da questão social e a prioridade colocada nos segmentos populacionais em situação de vulnerabilidade socioeconômica são algumas das evidências observadas. Mesmo sendo positivos os resultados obtidos, persistem rasgos do modelo residual e que se manifestam nas contradições do desenho, e também, na implementação de políticas sociais. Contudo, existem oportunidades para aprofundar as transformações em curso. O artigo apresenta, a partir de estudos empíricos e experiências na gestão a nível regional, alguns aportes para a análise de futuros cenários.

Palavras-chave: Políticas sociais. Esquerda. Estado.

\section{"Progressive" Social Policies in the Southern Cone: State discourses and practices}

Abstract: The change in orientation of social policies in Latin America, particularly in Mercosur, obeys the replacement of the political forces that have assumed the exercise of political power and control of the main resources of the state. The changes observed in the field of public policies in the direction of greater intervention of the state, a different approach to the social question and the priority given to population segments in a situation of socioeconomic vulnerability are some of the evidences observed. If results and successes are plausible, elements remain in the residual model that is manifest in contradictions in the design and implementation of social policies. Nevertheless, there are opportunities to deepen the transformations underway. The article presents some supports to the analysis of future scenarios, based on empiric studies and experiences in management at a regional level.

Keywords: Social policies. Left. State. 


\section{Introducción}

Al elaborar este artículo, estoy finalizando mi tarea en la conducción de un nuevo órgano regional dedicado especialmente a las políticas sociales - el Instituto Social del Mercosur - resultando un momento propicio para registrar y dar cuenta de las prácticas institucionales en la materia. Motivan las reflexiones y análisis expuestos, la necesidad de contribuir con una mirada crítica pero sin duda constructiva respecto de los avances, logros y metas obtenidas por los gobiernos progresistas o de izquierda en Sudamérica (aun cuando admitamos la existencia de matices significativos en términos de radicalidad de las respuestas estatales ante la cuestión social contemporánea), al mismo tiempo que aportar evidencias acerca de las insuficiencias, déficits y obstáculos a ser removidos para avanzar sustancialmente en dirección a la igualdad social y bienestar de las grandes mayorías latinoamericanas aun alejadas del beneficio del progreso tecnológico, científico y económico.

En un primer capítulo se explica brevemente desde el punto de vista metodológico, la concurrencia de dos fuentes fundamentales que proporcionan los elementos y argumentos que alimentan el análisis y examen de las políticas sociales aplicadas desde los inicios de la presente centuria. En el segundo capítulo se intentará dar cuenta - aportando una visión regional - de los discursos y las grandes definiciones en el campo del desarrollo social que se fueron generando desde los Estados, tomando básicamente los que integran el Bloque Mercosur, así como se explicitan las prácticas institucionales concretas y las políticas que se materializan en prestaciones, bienes y servicios públicos para la provisión del bienestar. En el tercer capítulo se colocan algunas de las contradicciones del actual modelo (bajo el supuesto que efectivamente hay evidencia suficiente para reconocer un conjunto de rasgos comunes que le confieren el estatuto de modelo), la secuela del paradigma liberal - residualista de los noventas y sus expresiones contemporáneas, las características emergentes de lo nuevo o lo innovador en el campo social y algunas claves para profundizar el proceso de cambios iniciado hace diez años en la Región. Finalmente, en el cuarto capítulo se esbozan (a modo de ensayo teórico) ciertas trayectorias por las cuales pudieran proyectarse el conjunto de actuaciones estatales asumiendo la conducción hacia un cambio radical del paradigma de inclusión e integración social en la Región.

\section{Abordaje teórico, las categorías conceptuales y los esquemas de interpretación (desde la investigación empírica y la experiencia de gestión)}

Como he señalado anteriormente, mis reflexiones se nutren - desde la perspectiva metodológica -, por un lado de mis observaciones y análisis de las decisiones y prácticas asumidas por los cuerpos burocráticos y los decision makers aplicados a la esfera del desarrollo social en la región y por el otro, de la investigación empírica y de manera comparada que he venido desarrollando a lo largo de cinco años en referencia a las políticas sociales emergentes en la última década en América Latina.

Con respecto a los propios estudios sobre las políticas sociales implementadas en la primera década del siglo 21 debe mencionarse el informe publicado por la Fundación Carolina, en marzo de 2010, en su serie Investigaciones N. 36, "Es posible un nuevo Estado de Bienestar en América Latina; la reconfiguración de las matrices de bienestar en el Mercosur" (MIRZA; LORENZELLI; BANGO, 2010), así como el estudio comparado relativo a las "Políticas Sociales de la Izquierda" en la región, junto a Carmen Midaglia y Alvaro Portillo (sin editar) del año 2011 y finalmente mi reciente trabajo para Clacso, (Re) Construyendo las matrices de bienestar, análisis comparado que consideró a Brasil, Bolivia, Venezuela y Uruguay (Mirza, 2013), actualmente en proceso de evaluación. Asimismo se incluyeron los valiosos aportes de Barba (2007), Martínez (2008) y Filgueira (2006). Por otra parte, la participación desde el año 2005 en los ámbitos del Mercosur Social, particularmente en la gestión de la dirección ejecutiva de un órgano de relativa reciente creación, como lo es el Instituto Social del Mercosur durante el período 2011 al 2013, me ha aportado una visión desde adentro y en contacto directo con los principales actores que producen decisiones, normas, estrategias y definen los itinerarios en la esfera del desarrollo social en sus respectivos países y a nivel regional.

a) Con relación a las categorías conceptuales utilizadas, las más importantes y recurrentes se desprenden del Libro hace poco publicado en Asunción, La Dimensión Social del Mercosur, marco conceptual (ISM, 2013), que remiten al enfoque de desarrollo social (superando la perspectiva exclusivamente económica); y refieren a la justificación de las nuevas políticas públicas centradas en la concepción de los ciudadanos como sujetos de derechos y no meramente como objetos de la asistencia pública; sustentando la vocación universalista de las intervenciones estatales (introduciendo y retomando la categoría de focalización subsidiaria); estableciendo la postulación de principios y criterios rectores de las políticas sociales, tales como la integralidad y el abordaje sistémico, así como la demercantilización del bienestar, desfamiliarización y desclientelización (ADELANTADO; SCHERER, 2008; ESPING-ANDERSEN, 1990). 
b) La explicitación de los conceptos manejados en el libro de referencia, se presenta como el resultado de un proceso complejo de discusión interna, en el cual participaron los investigadores provenientes del ámbito académico (a iniciativa de los ministerios de desarrollo social y del propio Instituto Social del Mercosur) los que fueron convocados en varias instancias para analizar el panorama de las políticas sociales en los respectivos países (aportando así algunos elementos importantes a la elaboración del libro); asimismo participaron durante más de dos años, técnicos y autoridades de desarrollo social de los respectivos ministerios, los que finalmente hubieron de dar el aval político a los contenidos finalmente elaborados por el equipo técnico del Instituto Social del Mercosur.

c) Por otra parte, cabe señalar que las múltiples reuniones, talleres de intercambio y sesiones de los diversos organismos que forman parte de la arquitectura institucional regional, particularmente concernientes a la dimensión social del Mercosur, han constituido una rica fuente de información para entender los procesos de construcción de políticas públicas y respuestas a las problemáticas sociales emergentes. En cierto sentido, la pluralidad de miradas y diversidad de experiencias exhiben tanto las coincidencias como las disonancias en el abordaje - por parte de los gobiernos - de la cuestión social y sus manifestaciones más evidentes. Fueron aquellos ámbitos en que se discuten y "negocian” acuerdos y resoluciones relativas a la dimensión social del Mercosur, una rica cantera de datos e informaciones que se convirtieron en insumos para el presente análisis.

Finalmente, cabe consignar que buena parte de este artículo incluye la ponencia presentada en el III Encuentro Estado y Políticas Sociales. Desafíos y oportunidades para el Trabajo Social latinoamericano y caribeño, coorganizado por Adasu (Uruguay) y la Federación Internacional de Trabajadores Sociales (FITS), celebrado en Montevideo en el pasado mes de mayo del 2013.

\section{Discursos y prácticas, emergentes y consolidadas, avances y limitaciones desde una perspectiva regional}

Los procesos de integración regional y muy particularmente el observado en el Mercosur, han tomado un giro hacia la identificación y jerarquización de dimensiones o esferas de lo social, entendidas como el campo de fuerzas en el que se desenvuelven las relaciones entre actores y sujetos portadores de intereses y demandas diversas, que procuran el bienestar para sí o los de su grupo (clases, corporaciones, colectivos de ciudadanos), y que disputan el poder y los recursos que lo garanticen.

La preocupación por atender enérgicamente las secuelas de las políticas neoliberales de fines del siglo 20, tales como el desempleo, la pobreza, la indigencia, la desnutrición infantil, la exclusión de millones de personas en situación de vulnerabilidad socioeconómica, entre otras consecuencias del pasado reciente y también como resultado acumulado desde varias décadas atrás, así como el empeño puesto en propender a la igualdad social, asemeja a todos los gobiernos autodefinidos de izquierda o progresistas. Los cambios en materia de políticas públicas que se vienen produciendo en el marco de unas economías de libre mercado, no dejan dudas respecto a la intencionalidad de cumplir con los compromisos declarados por los partidos políticos en sus respectivas plataformas electorales. No obstante, reconocer los impactos y logros obtenidos no implica ignorar las tensiones y contradicciones expuestas en la práctica, explicados en parte por factores externos y también por decisiones de políticas públicas no siempre congruentes con los discursos de las élites gobernantes.

Nuevas orientaciones en el abordaje de la Cuestión Social: en el devenir del último decenio se registraron cambios sustantivos en el campo de las políticas sociales en la Región, sobre todo a partir del acceso al ejercicio del gobierno de los partidos progresistas o de izquierda. En este sentido, el abordaje de la Cuestión Social manifiesta diferencias significativas respecto a los enfoques pasados al menos en dos puntos centrales. Por un lado se reconocen los derechos de ciudadanía y por otro, retorna el Estado Protector como gran regulador, articulador y proveedor del bienestar. Primera idea: el panorama de América Latina se reconfiguró radicalmente a partir de las conquistas electorales de los partidos y movimientos políticos que se ubicaban a la izquierda del espectro ideológico. Independientemente de cuán a la izquierda se encuentren, no cabe duda que la mayoría de los Estados están siendo gobernados por alianzas progresistas que se manifestaban como firmes opositores al modelo y a las políticas neoliberales implementadas en el último cuarto del siglo pasado. Desde la victoria de Hugo Chávez en Venezuela en 1999, pasando por las subsiguientes en Brasil, Argentina, Uruguay, Bolivia, Ecuador, Paraguay y Perú (solo teniendo en cuenta el subcontinente sudamericano), los partidos y sectores de izquierda o progresistas accedieron por la vía democrática liberal al ejercicio del poder político. Conviene subrayar que en Chile en los noventas, el poder político (tras la dictadura de Pinochet), estuvo en manos de la Concertación (alianza entre socialistas y democristianos), con resultados muy positivos en la disminución sensible de la pobreza pero incapaz de tocar la estructura de reparto de la riqueza. Más recientemente, los acontecimientos acaecidos en junio del 2012 en Paraguay significaron un claro retroceso respecto del tímido avance hacia un 
modelo más incluyente, iniciado por el ex presidente Fernando Lugo. El golpe parlamentario condujo a la reafirmación de la derecha oligárquica en el poder y como su corolario el resultado electoral del 21 de abril de 2013. Esta es sin embargo, la única excepción en el mapa del Mercosur.

Segunda idea: los abordajes de la cuestión social y la atención de sus múltiples y variadas manifestaciones fueron sustancialmente diferentes a las precedentes. Por un lado se reconocieron a los ciudadanos como portadores de derechos, es decir como sujetos y no objetos de la asistencia pública. Y por otro, el Estado antes prescindente o casi ausente, asumió un papel central en la construcción de respuestas de corto y medio alcance para enfrentar las secuelas dejadas por un enfoque mercado céntrico. Planes sociales de gran cobertura, sobre todo aplicados con un formato estándar de transferencias monetarias, que justo es recordar, se inicia mucho antes en México; políticas contundentes para reducir la pobreza y la extrema pobreza (con resultados plausibles); mayor intervención del Estado en la regulación de los mercados de trabajo; reformas sociales en curso, son apenas algunos ejemplos de los cambios ocurridos en la primera década de la presente centuria.

La institucionalidad regional emergente. No caben dudas que en cada Estado Nacional, particularmente los que constituyen el Mercosur, se verificaron transformaciones de las estructuras burocráticas de soporte, siendo relevante la creación de ministerios de desarrollo social, la consagración de dispositivos de control ciudadano, la formalización de espacios y órganos de coordinación de políticas públicas, tales que implicaron innovaciones efectivas en la ingeniería institucional del Estado Social. Y a nivel regional debe subrayarse la creación de ámbitos de articulación entre los Gobiernos, muy especialmente en el Bloque Mercosur y Unasur: Reunión de Ministros y Autoridades de Desarrollo Social (Rmads); Comisión de Coordinación de Ministerios de Asuntos Sociales (Ccmasm) y el surgimiento del Instituto Social del Mercosur (ISM) y del Consejo de Desarrollo Social en el segundo caso.

\section{El tiempo de recambio de los} gobiernos en el bloque del Mercosur pondrá en evidencia la solidez o fragilidad de las transformaciones realizadas en este primer decenio del siglo

\section{Las democracias se}

fortalecerán siempre que se asuman algunas deudas pendientes.
Tercera idea: el nuevo microcosmos institucional emergente pone en evidencia la prioridad política y programática de los gobiernos de la Región. La implementación de planes y programas dirigidos a la pobreza e indigencia, a la desnutrición, a la mortalidad materno infantil, entre otros problemas sociales exigieron la construcción de un cuerpo de organismos - con funciones específicas y complementarias - adecuado a los objetivos de la agenda social. La creación de los Ministerios de Desarrollo Social se justificó precisamente en la necesidad de dotar de instrumentos y dispositivos de intervención especializados en el combate a la pobreza y en la atención de situaciones de extrema vulnerabilidad socioeconómica (personas sin techo, hogares monoparentales con hijos a cargo, adultos mayores sin cobertura, desempleados de larga duración, niños y niñas con múltiples carencias, entre otros grupos sociales). De esa manera se aseguraría la eficacia de la acción estatal, al tiempo que la gestión de los recursos presupuestales suponía mayor eficiencia (focalización en cierto sentido) en la aplicación del gasto social.

Paralelamente, la instauración de ámbitos formalizados de concertación, coordinación y articulación de políticas públicas (ejemplos de Consejos Nacionales de Coordinación de Políticas Sociales y de Gabinetes Sociales liderados por la Presidencia en muchos casos), ilustra las reales motivaciones en el sentido de procurar intervenciones más sistémicas y menos fragmentadas.

Cuarta idea: La configuración de nuevas estructuras de articulación de políticas públicas a nivel de los Estados Nacionales se proyecta a nivel regional en ámbitos institucionalizados. En este sentido se destaca - como se ha dicho - la creación de la Reunión de Ministros y Autoridades Sociales del Mercosur (Rmads) y del Consejo de Desarrollo Social de Unasur. La creación en el año 2007 del Instituto Social del Mercosur (ISM) se corresponde con la emergencia de la dimensión social de la integración regional con la intención de dotar de un instrumento técnico político capaz de asesorar y asistir a los diversos organismos con competencias en el área de las políticas sociales. La consolidación de los Grupos y Redes Especializadas (Empleo, Género, Infancia, Educación), confirman esta tendencia de coordinación e intercambio entre Estados y la viabilización de acuerdos de cooperación bi y multilaterales.

Una agenda social común: por otra parte, la similitud de enfoques, propuestas y políticas aplicadas en la Región condujo de alguna manera a construir una agenda social común, plasmada en el Plan Estratégico de Acción Social (PEAS; ISM, 2012), estableciendo las prioridades, objetivos y compromisos en el campo del desarrollo social en el Mercosur. 
Quinta Idea: en efecto, el PEAS se constituye como una hoja de ruta indicativa acerca de las metas y objetivos sociales, comunes y consensuados en la Región. Dicho Plan está vertebrado en 10 ejes, 26 directrices estratégicas y 101 objetivos prioritarios que comprometen a los Estados que lo suscribieron en junio del año 2011 en la Cumbre de Presidentes realizada en Asunción. La agenda social cobra entonces la mayor legitimidad política a nivel subregional y se traslada al ámbito más amplio de Unasur que se encuentra actualmente en debate para la elaboración de su respectivo Plan Estratégico en la órbita social:

El PEAS contiene indicaciones y objetivos específicos en lo referido a: 1) Erradicar el hambre, la pobreza y combatir las desigualdades sociales. 2) Garantizar los derechos humanos, la asistencia humanitaria y la igualdad étnica, racial y de género. 3) Universalizar la salud pública. 4) Universalizar la educación y erradicar el analfabetismo. 5) Valorizar y promover la diversidad cultural. 6) Garantizar la inclusión productiva. 7) Asegurar el acceso al trabajo decente y a los derechos de previsión social. 8) Promover la sustentabilidad ambiental. 9) Asegurar el diálogo social. 10) Establecer mecanismos de cooperación regional para la implementación y el financiamiento de políticas sociales (ISM, 2012, p. 41-74).

Tendencias convergentes. Al examinar las características que fueron reflejando las estrategias y políticas públicas aplicadas hallamos grandes coincidencias en casi toda la Región. Si las administraciones progresistas implementaron mecanismos de reversión de las tendencias de pauperización a través de diferentes planes y programas de transferencia monetaria, que se acoplaron a la estructura de protección social preexistente, existe un terreno fértil para "radicalizar" la estrategia de combate a la pobreza y profundizar el camino hacia la igualdad social. Asimismo en casi todos los casos identificamos iniciativas de reformas sociales que, aun cuando no son completas y profundas, constituyen avances importantes en cuanto a la reconfiguración de la matriz de bienestar y auguran en principio mejoras sustantivas en la calidad de vida de las poblaciones.

Sexta Idea: Existe evidencia suficiente para demostrar que hay un modelo de desarrollo social que es compartido por la enorme mayoría de las naciones sudamericanas. Dichos rasgos comunes y convergentes se resumen en los siguientes puntos: a) retorno del Estado protector y promotor de derechos; b) integralidad de las políticas públicas; c) se inclina la balanza hacia la universalidad; d) focalización subsidiaria de las políticas sectoriales; e) transferencia condicionada de renta en expansión; f) incremento de la Inversión Social (Gasto Público Social incluso con carácter contracíclico); g) reformas sociales en curso (básicamente en educación y salud); h) relevancia de la articulación intersectorial e innovaciones institucionales; i) políticas sociales y políticas económicas no contrapuestas; j) perspectiva territorial y descentralización; k) promoción de mecanismos de control y participación ciudadana.

En el Libro citado se enumeran los principios y criterios fundamentales que deben orientar las políticas sociales progresistas:

a) Centralidad de la dimensión social de la integración que pretenda promover un desarrollo humano y social integral; b) Indisociabilidad de lo social y económico en la formulación, el diseño, la implementación y la evaluación de las políticas sociales regionales; c) Reafirmación del núcleo familiar como eje de intervención privilegiado de las políticas sociales en la Región; d) Relevancia de la seguridad alimentaria y nutricional; e) Centralidad del rol del Estado; f) Protección y promoción social desde una perspectiva de derechos, superando la visión meramente compensatoria de lo social; g) Transversalidad de un enfoque dirigido a contrarrestar y evitar todo tipo de discriminación, sea por cuestiones étnico-raciales, de género o generacionales; h) Participación comunitaria, sociedad civil fortalecida organizacionalmente y i) Perspectiva territorial y descentralizada de las intervenciones públicas, considerando la reducción de las asimetrías intra y transfronterizas (ISM, 2013, p. 20-21).

Por otra parte, si bien se reconocen avances sustantivos en una variada y amplia gama de indicadores sociales, desde la tasa de pobreza y de indigencia hasta la tasa de desempleo pasando por la reducción sensible de la tasa de mortalidad infantil, todos los datos evidencian claramente la eficacia de las políticas sociales, más no pueden evitar la reproducción de la pobreza al margen de las intervenciones estatales. La propia estructura de producción, distribución y consumo responde a un patrón capitalista, que de hecho se constituye como un freno que las políticas de protección social no pueden absorber. La pobreza desciende, pero no implica necesariamente su eliminación en tanto se mantengan los clivajes de la acumulación de capital, en otras palabras, las restricciones y límites de las políticas sociales de cuño progresista están demarcados por el propio sistema económico. 


\section{Nova et véttera: el lastre de lo mismo y las claves del cambio}

Si efectivamente hubo un avance sustancial en muchos indicadores del bienestar en la Región, por otra parte persisten problemas estructurales que no han podido ser superados, sobre todo si tomamos nota de la distribución de la riqueza y la situación de vulnerabilidad socioeconómica en la que se hallan aún centenas de miles de ciudadanos en la Región. Desde luego que, al examinar los discursos y las propuestas electorales y las comparamos con las políticas aplicadas, identificamos algunas contradicciones que se reconocen como obstáculos reales a ser superados.

Primera contradicción: en el discurso se coloca el fundamento ético y político en el ciudadano como portador de derechos inherentes al ser sujeto. Sin embargo la mayoría de los programas más emblemáticos, tales como los de transferencia monetaria directa a los sectores sociales más vulnerables, siguen la pauta de las condicionalidades o de la corresponsabilidad. Vale decir que, al reconocimiento de un derecho, se le adiciona una condición para ejercerlo. Si los ciudadanos que son destinatarios de las prestaciones económicas no cumplen con lo estipulado por el programa, generalmente - pero no únicamente - sujeto a la concurrencia a los centros de salud y a los servicios educativos básicamente a nivel escolar, entonces no habrán de recibir aquella prestación en dinero.

Segunda contradicción: en el discurso se hace hincapié en el criterio universalista de las políticas sociales, lo que supone ningún tipo de discriminación positiva o selección ex ante de los destinatarios de aquellas. No obstante, la focalización es el criterio utilizado mayormente por innumerables programas asistenciales. Convengamos que, son a cierto punto y grado, conciliables y compatibles en función de la disponibilidad de recursos fiscales y fundados en la necesidad de lograr eficacia y eficiencia. Pero, en planes de cobertura masiva, nuevamente en el caso de las transferencias monetarias, se focaliza la población con criterios técnicos más o menos objetivos, pero inevitablemente quedan colectivos fuera de la mentada focalización con frecuencia por factores difíciles de interpretar. Muchos hogares quedan al borde o en la frontera, porque no se categorizan como sectores pobres, siendo que permanecen en la franja de vulnerabilidad y expuestos a las oscilaciones de ingresos que eventualmente los hará caer en situación de pobreza.

Tercera contradicción: cuando se convierten derechos en obligaciones se subvierte el concepto de ciudadanía. En efecto, es el caso de los programas de trabajo protegido o promovido (de tipo workfare) que con el supuesto de facilitar la reincorporación de los desempleados crónicos al mercado laboral establece contraprestaciones a los subsidios. En ocasiones, se obliga a realizar determinados trabajos comunitarios a cambio de recibir lo que incluso jurídicamente no se reconoce como empleo temporario, con el consiguiente desconocimiento de los derechos laborales. Por consiguiente, el derecho al trabajo se convierte en obligación de trabajar para obtener una prestación económica a cambio.

Cuarta contradicción: se proclama la prioridad el combate a la pobreza y la exclusión, sin embargo la afectación de los recursos del Estado a los planes de transferencia monetaria directa no superan prácticamente el $1 \%$ del PBI (a excepción de Ecuador), siendo los montos exiguos en función de las necesidades y demandas de los hogares más vulnerables. Claro está, igualmente el Gasto Público Social expresa la medida de las prioridades en el bienestar de la población. Y este indicador ha progresado notablemente en los últimos diez años, superando el $24 \%$ del PBI como promedio del Bloque y cerca del $18 \%$ para el subcontinente.

Si estas contradicciones son apenas algunas de las más evidentes, el proceso complejo de superación parte en primer lugar de su identificación como tales y en segundo lugar, de repensar las estrategias adecuadas en contextos no siempre favorables, en los que se conjugan limitaciones materiales y simbólicas. Las primeras derivadas de la racionalidad financiera y fiscal, las segundas como expresiones culturales e ideológicas que permean todas las clases sociales y todo el espectro político. Aun con un Estado protector, regulador y promotor de los derechos sociales, buena parte de las sociedades contemporáneas, mantienen convicciones y creencias respecto a la pobreza y la exclusión, en cierto sentido, conservadoras y regresivas: el pobre debe ser merecedor de la provisión de subsidios, y de una asistencia pública sostenida por los ciudadanos contribuyentes y por ende, difícil será encontrar argumentos convincentes para abandonar la focalización, las condicionalidades de las transferencias monetarias o implementar medidas más radicales en el sentido redistributivo de la riqueza. Las claves de los cambios más profundos y necesarios podrían estar en la dimensión cultural por un lado, pero asimismo en la osadía de las nuevas generaciones al mando de los estamentos estatales y de las élites progresistas que hoy ejercen el poder político y controlan buena parte de las decisiones y orientaciones de política pública.

\section{Utopías, políticas y política}

Al analizar el panorama político, económico y social en la Región y proyectando escenarios futuros de medio plazo, los cambios parecen consolidarse en términos generales y más allá de situaciones particulares en 
el Cono Sur. En suma, en mayor o menor medida, con los riesgos latentes y las debilidades reconocidas, se están construyendo nuevas Matrices de Bienestar. El tiempo de recambio de los gobiernos en el bloque del Mercosur pondrá en evidencia la solidez o fragilidad de las transformaciones realizadas en este primer decenio del siglo 21. Las democracias se fortalecerán siempre que se asuman algunas deudas pendientes.

Séptima Idea: Las deudas pendientes pasan por abordar con coraje la enorme desigualdad social aún persistente en la Región. Se reconocen los avances importantes que se han traducido en la disminución de la brecha social medida por el Gini, y desde luego en la drástica reducción de las tasas de indigencia y de pobreza, también en la regulación del mercado de empleo y sus efectos beneficiosos para la clase trabajadora. No obstante, en el marco de una bonanza económica que se ha expresado en altas tasas de crecimiento del PBI, la desigualdad exige imaginar y diseñar opciones de alto impacto. Junto con el examen de alternativas universales de transferencia de renta, tales como la implementación de un Ingreso Ciudadano Universal e Incondicional o la universalización de prestaciones en el polo no contributivo (ejemplo Asignaciones para menores de 18 años y para mayores de 65), será imprescindible profundizar o encaminar reformas tributarias (en algunos países ya iniciada), tales que generen una redistribución equitativa de la riqueza.

Los gobiernos progresistas al mando de los Estados poseen condiciones para reinventar utopías, los dirigentes políticos de izquierda tienen delante de sí una oportunidad inmejorable para avanzar hacia escenarios de mayor igualdad social y echar el lastre de las décadas pasadas (sobre todo desprenderse de los rasgos del modelo liberal residualista), que pesa en el diseño e instrumentación de políticas integradoras e inclusivas. Los Estados son campos de disputa, por lo que tanto es importante el diseño sustantivo de estrategias y políticas innovadoras como la transformación de los aparatos burocráticos y su lógica procedimental racionalista. Más aún, el Estado al servicio de la ciudadanía debe tener en cuenta la posibilidad de incorporar con mayor vigor la participación social en sus propias estructuras operativas. En este sentido se pueden observar avances importantes, tanto en términos de una descentralización política y administrativa, como en la instauración de mecanismos de contralor ciudadano. Centros de referencia territorial, mesas locales y consejos sociales son algunas de las modalidades adoptadas en la Región, lo que ha generado una densidad organizacional expresada en múltiples ámbitos de intercambio y retroalimentación para mejorar la aplicación de políticas sociales en mejor consonancia con las necesidades y demandas de la población.

No obstante, la política siendo el locus preferencial de la confrontación, el diálogo y la negociación, allí donde se manifiestan los conflictos sociales. En tanto se mantengan los clivajes del sistema capitalista con su lógica de acumulación, el terreno de oposición y conciliación de intereses se dirime democráticamente bajo reglas de juego específicas y condicionadas por la lógica del capital. Hay aquí un campo propicio para dar un salto cualitativo, estableciendo o expandiendo modalidades participativas, tales como los mecanismos de consulta plebiscitaria, los referéndums y las asambleas barriales. El libreto democrático admite indagar y ensayar nuevas opciones orgánicas e institucionalizadas de participación popular sin menoscabo de las formas tradicionales de la democracia liberal.

\section{Algunas conclusiones}

La variable política explica en gran medida las transformaciones en la orientación de las políticas sociales en la Región; tanto por sus objetivos, así como por sus modalidades instrumentales. La combinación de planes de atención de las situaciones de pobreza e indigencia, con procesos de reformas sociales ha dibujado escenarios de reconfiguración de las matrices de bienestar, en tanto se registran tendencias sostenidas hacia la desmercantilización y fuerte presencia del Estado en la provisión de prestaciones y servicios sociales para el pleno ejercicio de los derechos de ciudadanía. Pero hace falta remover los rasgos del modelo liberal - residual aún presentes en casi toda la Región. Superar las contradicciones expuestas implica romper con algunas tradiciones de política social, particularmente aquellas que responsabilizan a los individuos por su situación de vulnerabilidad y pobreza, sean aquellas tácitamente aceptadas o normativamente estipuladas.

Existe convergencia estratégica y normativa en la región, que culmina en la elaboración consensuada del más alto nivel político de una agenda social común al menos para la zona Mercosur. Asimismo la ingeniería institucional en Sudamérica manifiesta el reconocimiento de la otra cara de la integración regional, no limitada exclusivamente a los dominios de la regulación comercial, aduanera y arancelaria. Sin embargo la decisión de impulsar un Plan Estratégico de Acción Social sin recursos financieros puede acabar en mera fantasía. No hay política pública sin recursos públicos. Empero, debe subrayarse que los abordajes de la cuestión social, siendo muy similares entre los Estados, son un factor que agrega fortaleza a cada uno y produce sinergia en el conjunto de los miembros de la comunidad regional.

La voluntad y el discurso de inclusión-integración es plausible, pero no basta. Las contradicciones del sistema económico ponen al descubierto las limitantes estructurales que los sistemas de protección social no 
pueden absorber. Sin una revisión profunda de los modelos de producción, distribución y consumo las políticas sociales encontrarán los límites de sus efectos redistributivos. Consecuentemente, cuando se proyectan escenarios a diez o veinte años, no se trata de extrapolar a futuro las variables más significativas que hacen al bienestar social en función de las expectativas económicas asociadas a las tasas de crecimiento del PBI, al grado inversor, o el mantenimiento de cierta estabilidad macroeconómica. Esta postura o perspectiva es digámoslo así, conservadora.

Es necesario construir fuertes hegemonías en América Latina. Los intereses de clase trascienden las fronteras jurisdiccionales de los Estados Nacionales. Los grandes grupos y corporaciones económicas que disputan el control de los clivajes del poder no dan tregua. Si los partidos políticos de izquierda hoy en el ejercicio del poder político no robustecen y amplifican las alianzas con los movimientos y actores sociales de la Región, los avances y logros obtenidos - que han sido enormes por cierto - podrían ser revertidos a futuro. Las políticas sociales, es bien sabido, son el reflejo o el resultado de conquistas y negociaciones entre partes en conflicto. Las alianzas tejidas entre actores políticos y actores sociales - en más de diez años en algunos casos - son fundamentales para sostener los avances y logros obtenidos en los sistemas de protección social; para profundizar y corregir sus aspectos regresivos serán trascendentes pactos de mayor envergadura y compromisos por la equidad e igualdad social. Pacto fiscal o pacto social exigen entre otros requisitos, una disminución de la presión impositiva al Trabajo y un aumento de la presión tributaria al Capital.

La oportunidad histórica que tiene la Región, exige aprovecharla para que el tránsito de un modelo excluyente o dual, a un paradigma incluyente y equitativo sea irreversible. ¿Es posible construir un nuevo Estado de Bienestar en la Región? No nos referimos a aquel producto socio histórico europeo - hoy convertido en una caricatura sino a un modelo propio, latinoamericano, construido sobre la base de aprendizajes, de contradicciones, de interrogantes e incertidumbres. De ello también dependerá la suerte de las democracias latinoamericanas.

Generar espacios de debate político en torno a proyectos societarios y nuevas utopías será primordial, en los tiempos venideros los sectores conservadores y las élites económicas de la Región seguirán en su afán por recuperar los beneficios y privilegios que otrora les eran otorgados por gobiernos y Estados por ellas capturados, e inevitablemente asociados a políticas sociales apenas compensatorias, magras y degradadas en su función redistributiva e incluyente. Que los Estados garanticen el ejercicio pleno de los derechos de ciudadanía comporta una buena dosis de audacia, compromiso y firmeza en los principios y postulados ético-políticos, todo lo cual halla en la coyuntura contemporánea un terreno más que fértil.

\section{Referencias}

ADELANTADO, J.; SCHERER, E. Desigualdad, democracia y políticas sociales focalizadas en América Latina. Revista Chilena de Administración Pública, Santiago, v. 11, p. 117-134, 2008.

BARBA, S. C. La nueva cuestión social en el Mundo y América Latina: más allá de la pobreza. Revista Renglones, Guadalajara, v. 62, p. 25-44, 2010.

ESPING-ANDERSEN, G. Three Worlds of Welfare Capitalism. Cambridge: Polity, 1990.

FILGUEIRA, F. El nuevo modelo de prestaciones sociales en América Latina, eficiencia, residualismo y ciudadanía estratificada. En: ROBERTS, B. Ciudadanía y política social latinoamericana. Costa Rica: Flacso/SSRC, 1998.

ISM-Instituto Social del Mercosur. Plan Estratégico de Acción Social. Asunción, 2012. Disponible en: <www.ismercosur.org>. Acceso en: 7 jul. 2013.

. Marco conceptual de la dimensión social. Asunción, 2013. Disponible en: <www.ismercosur.org〉. Acceso en: 7 jul. 2013.

MARTÍNEZ, J. Regímenes de bienestar en América Latina: ¿cuáles y cómo son? Madrid: Fundación Carolina - CeALCI, 2008.

MIRZA, C. A.; LORENZELLI, M.; BANGO, J. ¿Es posible un nuevo Estado de bienestar en América Latina?: la reconfiguración de las matrices de bienestar en el Mercosur. Madrid: Fundación Carolina - CeALCI, 2010.

Christian Adel Mirza

cmirzap@gmail.com

Mestre em Ciência Política pela Universidad de la República (Udelar)

Professor do Departamento de Trabajo Social. Facultad de Ciências Sociales da Udelar

\section{Udelar}

Constituyente 1502

Montevideo - Uruguay

CEP: 11200 